\title{
A Brief Analysis of the Properties and Characteristics of Cultural and Creative Products
}

\author{
Qiku Bao ${ }^{1}$ and Mo Wang ${ }^{1}$ \\ ${ }^{1}$ Jilin Animation Institute, Changchun, Jilin
}

Keyword: Cultural and creative products;Properties; Characteristics

\begin{abstract}
With the continuous development of social civilization, cultural and creative products have come to impact people's life with their own advantages.
\end{abstract}

\section{Introduction}

This paper mainly introduces the related concepts, the composition and classification and primary properties of cultural and creative products, based on which to analyze fundamental characteristics cultural and creative products have so as to better promote the development.

\section{Definition of the Related Concept}

Definition of Culture. Culture, in a relatively broad sense, can be regarded as the sum of material and spiritual wealth humans produce. While Culture with narrow definition, in a sense, can be seen as the sum of spiritual wealth humans create. For the latter, it mainly refers to the intellectual activities humans take on, and thereby the relevant achievements produced. Most widely, it is generally what is called scientific and technological achievements including copyright and related patents etc. and also the corresponding spirit quality humans have.

Definition of Creativity. Creativity is closely tied to the words like innovation and culture but from the main level Creativity innovation cannot be exactly the same as equal to innovation and then not equal to culture. In a certain sense, Innovation has a certain degree of spontaneity, not on the basis of human's consciousness and does not alter either as it changes. To a large extent, creativity is the study of subjective initiative of humans, with the main focus on the development and utilization of humans intelligence, and it is produced through a certain degree of creative power with the relative imagination of humans. And therefore it is a further innovation of subjective consciousness of research contents For creativity itself, to some extent, it is hard to conduct a proper argumentation in the strict sense. By contrast, innovation is a sort of conscious creative activities.

Cultural and Creative Products. Cultural and creative products can be regarded as the products cultural and creative industries produce in the course of its development in spite of forms and combinations of products.From the view of the shape the main products can present ultimately, cultural and creative products comprise two important components. One is creative content the other is corresponding hardware carrier. The relationship between the two is both interdependent and mutually developed. cultural and creative products are different from other products mainly in the respect of cultural and creative contents they convey which are also their major core values. Specificity of cultural and creative products also requires that cultural and creative contents are unable to exist independently by themselves and need to lean on major hardware carriers.

\section{Composition and Classification of Cultural and Creative Products}

Composition of Cultural and Creative Products. With the continuous development of modern life, cultural and creative products can be roughly split into three major parts, which are produce of main spiritual products, produce of pure spiritual products and produce of the corresponding general spiritual products respectively. The most important among them are produce of pure spiritual products which are mainly based on cultural and creative contents as the corresponding activities. Produce of spiritual products can be seen as the produce 
of pure spiritual products, which is mainly for the purpose of providing corresponding activities that have applicability and cultural creativity and so on for the various relevant material products. Among which, produce of pure spiritual products is the main core part of the research on the whole cultural and creative industries.

Classification of Cultural and Creative Products. Cultural and creative products can be classified to two main parts, which need to undergo two main processes of development. The two processes are industrialization of creativity and creativity of industries. So cultural and creative industries can be divided into two types which are consumptive type and productive type respectively. For the former type, they are indispensable for forming main industrialization of creativity and focus on the whole process of creativity compared to productive type cultural and creative industries. In a word cultural and creative industries are core component of the researched industries. During the research, Not only should the overall consumption of cultural knowledge be enhanced but on this basis should the related application of cultural knowledge and effective innovation be further stressed.

\section{Major Properties of Cultural and Creative Products}

Value Properties of Cultural and Creative Products. Value properties of cultural and creative products refer to the entirety of human's mental activities and corresponding effects produced which are expressed in a relatively cultural and creative product. Value properties can also be seen as relative statutory specificity that Value ownership has and also can be referred as uniqueness of ownership which just means protectiveness of intellectual property, namely the protection of creative achievements. That is to say that from the ascription of creative achievements, overall creativity is owned by individual and is effectively protected by laws and other people do not possess it in a position. It can be considered from the level of the overall intellectual property that uniqueness of creative products is fixed mainly according to relevant laws. And it can be seen intellectual property possess the statutory specificity. In Hoggins' view, Creative Economy by him gives detailed definition to creative products. Creative products can be regarded as referential economic products protected in the scope of relevant intellectual property.In his book, he deems that intellectual property can be classified to 4 types namely patents, copyrights, trademarks and the overall design. For his study, the specific classification has relevant industrial counterparts which are important parts of creative industries and economy.In other words, creativity industries can be regarded as the economic category of corresponding intellectual property in a sense. That is to say creativity would not have had greater values without the development of creativity industries.

Economic Property of Cultural and Creative Products. To some extent the mode of presence of cultural and creative products is price them appropriately and market them and then turn incorporeal capital to actual money and form values accordingly, which thereby can drive indirect and relatively direct economic growth and moreover boost employment growth accordingly. The sum these economies bring about is the concerned economic value overall cultural and creative products can produce.

\section{Main Features of Cultural and Creative Products}

The Two Properties of Culture and Innovation. As for cultural creativity, it has double complexities. As the name implies, it integrates culture into innovation. For itself, innovation is its essential property. Cultural creativity is different from cultural innovation and more different from so called culture. So cultural innovation is an extension of culture and innovation and synthesis of culture and innovation. So it has the dual properties of culture and innovation. Creativity industries can be regarded as innovative industries. The main research process is aimed at the whole creative production and the whole process of corresponding marketing. In other words, innovation cannot be regarded as traditional invention but targeted reconfiguration of utilizable resources, and the process of development of creativity. Under this mode of thinking, it can be widely utilized and accepted effectively and then gets recognized wholly on the ideological level and furthermore achieve economic benefits in the overall running of the market. 
High Penetrability. For cultural creativity itself, it is intangible resource. For creative people, it is an important part that conceives new thoughts and ideas. From the view of whole cultural creativity industries, The main part of creativity is human while the sources of creativity are based on human's wisdom and the major basis of creativity is based on basic perception and knowledge accumulation. Humans can develop creativity further only via the constant development of relevant creative imagination. All in all, cultural creativity has affected multiaspects and displayedits proper values.

Low Pollution, Consumptive. Cultural products are less dependent on principal natural resources and thus have the nature of low pollution and low consumption and occupy less space. The main features of cultural products are realize digital and networked consumption and less dependent on the surrounding resources. The traditional industries are mainly labor intensive industries centered on processing and manufacturing. In contrast, cultural creativity industries are different from tradition with a focus on the research of knowledge intensive and mostly are centered on mental work and focus the core of research on the crystallization of human's wisdom of creativity. Human's creativity possesses intangibility and can be regarded as renewable and even inexhaustible. The development of creative products is not limited to the external limitations any longer and meanwhile does not damage relevant industries. On the contrary it promote the development of various industries and bring about higher added values.

Non-competitiveness and Non-exclusiveness. In contrast to most actual things, the forms of creative products possess non competitiveness and non exclusiveness to some extent. Non-competitiveness can be seen as the consumption of the certain products that some people conduct without an impact on the effective consumption of the same products other people conduct. The benefits that some achieve from the product do not affect the benefits that others get from the product. So it is clear that the radical conflict of interest does not exist between beneficiaries in reality. For the study on the consumption of cultural and creative products, intensive study needs to be conducted aimed at the nature of non-competitiveness and non-exclusiveness and promote impactful means and measures through appropriate practices and make a targeted response combined with related technologyavoiding the effect and damage caused.

Possessing Higher Resilience of Demand. For the understanding of concept, resilience, widely used in economics, mainly refers to the overall extent of reaction of dependent variables when independent variables alter. Resilience of demand can be seen as being used for measurement and it can measure the overall extent of reaction of the quantity of a demanded product as some certain demanded determinants change, or to be more exact and specific, resilience of demand is the right measurement of the percentage of change of quantity demanded (dependent variables)caused as the values of demanded determinants change by $1 \%$. Resilience of demand can be divided into 2 parts including income resilience of demand and price resilience of demand. Income resilience of demand mainly refers to due to the change of income.

\section{Conclusion}

Cultural and creative products have increasingly impacted people's daily life. Only the enhancement of the understanding of their properties and characteristics can promote the development of creativity better. With the development and change of the times, the development of and emphasis on cultural and creative products should be enhanced. The development of creativity of cultural industries is promoted while meeting people's daily demands.

\section{References}

[1] P.Z.Wei.2010.The introduction to cultural and creative industries. Renmin University of China Pres.

[2] Y. Zhao. 2004. Option pricing theory and investment decision analysis under uncertainty. China Agriculture University Press.

[3] S.F.Han .2010. Research on the value of creative industry based on two directions. Progress of science and technology and Countermeasures. (4) : 60-63. 
[4] Z.B.Wang. 2008. Analysis of factors influencing the cultural products. Journal of zhongnan university of economics and law. (5):120-123.

[5] H.T and J.Z. 2011. Research on consumer perceived value of cultural products. Journal of Southwest University for Nationalities: Humanities and Social Sciences. (11):136-140.

[6] X.Q.Zang and T.You. 2011. Cultural products: recognition features and attributes. Discovery (5):120-123.

[7] P.Z.Wei.2010. The attributes and characteristics of cultural and creative products. Culture Monthly. (8):54-58. 
